December 10, 2018 1:44 WSPC/INSTRUCTION FILE altnew

\title{
Constraints on a new alternative model to dark energy
}

\author{
Yungui Gong \\ Institute of Applied Physics and College of Electronic Engineering, Chongqing University of \\ Post and Communication, Chongqing 400065, China \\ gongyg@cqupt.edu.cn \\ Xi-Ming Chen \\ College of Electronic Engineering, Chongqing University of Post and Communication, \\ Chongqing 400065, China
}

\begin{abstract}
The recent type Ia supernova data suggest that the Universe is accelerating now and decelerated in recent past. This may provide the evidence that the standard Friedmann equation needs to be modified. We analyze in detail a new model in the context of modified Friedmann equation using the supernova data published by the High- $z$ Supernova Search Team and the Supernova Cosmology Project. The new model explains recent acceleration and past deceleration. Furthermore, the acceleration of the expansion of the Universe is almost zero in the future.
\end{abstract}

Keywords: Dark energy; Alternative model; type Ia supernova.

\section{Introduction}

The type Ia supernova (SN Ia) observations support that the expansion of the Universe is speeding up rather than slowing down ${ }^{1,2,3}$. A spatially flat universe is favored by the measurements of the anisotropy of the cosmic microwave background $(\mathrm{CMB})^{4,5}$. The observation of type Ia supernova SN $1997 \mathrm{ff}$ at $z \sim 1.7$ also provides the evidence that the Universe is in the acceleration phase and was in the deceleration phase in recent past ${ }^{6,7}$. The transition from the deceleration phase to the acceleration phase happens around the redshift $z_{\mathrm{T}} \sim 0.4^{7,8}$. The simplest way of explaining the acceleration phenomenon is through the cosmological constant with the equation of state parameter $\omega_{\Lambda}=-1$. One easily generalizes the cosmological constant model to dynamical cosmological constant models, such as the dark energy model with negative constant equation of state parameter $-1 \leq \omega_{\mathrm{Q}}<-1 / 3$ and the holographic dark energy models ${ }^{9,10}$. In general, a scalar field $Q$ called quintessence field that slowly evolves down its potential $V(Q)$ takes the role of a dynamical cosmological constant ${ }^{11,12,13,14}$. The energy density of the quintessence field must remain very small compared with radiation and matter at early epoches and evolves in a way that it started to dominate the Universe around the redshift 0.4 . If we remove the null energy condition restriction $\omega_{\mathrm{Q}} \geq-1$ to allow supernegative $\omega_{\mathrm{Q}}<-1$, then the phantom energy models arise ${ }^{15}$. More exotic equation of 
state is also possible, such as the Chaplygin gas model with the equation of state $p=-A / \rho$ and the generalized Chaplygin gas models with the equation of state $p=-A / \rho^{\alpha 16}$. There are fields other than the quintessence field, such as tachyon field, as dark energy ${ }^{17}$.

Although there are many dark energy models proposed in the literature, the nature of dark energy is still unknown. Therefore it is also possible that the observations show a sign of the breakdown of the standard cosmology. In brane cosmology, our observable universe is a 3-brane which is located at the $Z_{2}$ symmetry fixed plane of a five dimensional Ads (Anti-de Sitter) spacetime with the extra dimension to be an orbifold $S_{1} / Z_{2}$. Binétruy, Deffayet and Langlois showed that the Friedmann equation takes the unconventional form $H^{2} \sim \rho+\rho^{2}$ in brane cosmology ${ }^{18}$. In addition, Chung and Freese argued that almost any relationship between $H$ and $\rho$ was possible with the help of extra dimension ${ }^{19}$. Along this line of reasoning, the usual Friedmann equation $H^{2}=8 \pi G \rho / 3$ is modified to a general form $H^{2}=g(\rho)$ $20,21,22,23,24,25,26$. In these models, no exotic matter form is needed and the Universe is composed of the ordinary matter and radiation only. The function $g(x)$ satisfies the following conditions: $(1) g\left(x_{0}\right)=1$; (2) $g(x) \approx x$ when $z \gg 1$; (3) $g\left(x_{0}\right)>3 x_{0} g^{\prime}\left(x_{0}\right) / 2$, where $x=\Omega_{\mathrm{m} 0}(1+z)^{3}+\Omega_{\mathrm{r} 0}(1+z)^{4}, x_{0}=\Omega_{\mathrm{m} 0}+\Omega_{\mathrm{r} 0} \approx \Omega_{\mathrm{m} 0}$ and $\Omega_{\mathrm{r} 0}=8.40 \times 10^{-5} 5$. In Cardassian model, $g(x)=x+B x^{n}$. In generalized Cardassian model, $g(x)=x\left[1+B x^{\alpha(n-1)}\right]^{1 / \alpha} 20$. For Chaplygin gas like model, $g(x)=x+\left(A^{\prime}+B^{\prime} x^{\beta}\right)^{1 / \beta} 25$. In Ref. 21, Friedmann equation is modified to be $H^{2}+H^{\alpha} \sim \rho$ based on the idea of embedding a three brane into a five dimensional flat spacetime. The modified model can be mapped into dark energy model. For example, $g(x)=x$ for standard cosmology; $g(x)=x+\Lambda$ for $\Lambda$ CDM model; $g(x)=x+B x^{1+\omega_{\mathrm{Q}}}$ for dark energy models with constant equation of state parameter $\omega_{\mathrm{Q}}$. In this paper, we discuss the new model proposed in Ref. 26 in detail. We first derive the model with the help of extra dimension, then we use different SN Ia data sets to fit the model.

\section{Model Setup}

Follow Chung and Freese ${ }^{19}$, the new model motivated by extra dimensions can be derived from the action

$$
S_{5}=\frac{-1}{2 \kappa_{5}} \int d^{5} x \sqrt{\mathcal{G}} \mathbb{R}+S_{\text {orb }}+S_{\text {boundary }},
$$

where $\mathbb{R}$ is the Ricci scalar in five dimensions, $\mathcal{G}$ is the five dimensional metric determinant, $\kappa_{5}=1 / m_{p l, 5}^{3 / 2}$ is the five dimensional Newton's constant, $S_{\text {orb }}$ is the orbifold or the bulk action, and $S_{\text {boundary }}$ represents the boundary or the orbifold fixed plane action. In this setup, our universe is a 3-brane residing at the boundary of the fifth orbifold $S_{1} / Z_{2}$. Varying the above action with respect to the metric, we get the five dimensional Einstein equation

$$
G_{M N}=\mathbb{R}_{M N}-\frac{1}{2} g_{M N} \mathbb{R}=\kappa_{5}^{2} T_{M N}+\kappa_{5}^{2} t_{M N} \delta(\Sigma),
$$


where $T_{M N}$ is the energy momentum tensor of the bulk and $t_{M N}$ is the energy momentum tensor of the boundary.

To get cosmological solutions, we take the general five dimensional metric for an isotropic and homogeneous flat brane universe embedded in five dimensions as

$$
d s^{2}=-N^{2}(t, y) d t^{2}+a^{2}(t, y) \delta_{i j} d x^{i} d x^{j}+B^{2}(t, y) d y^{2} .
$$

The components of the five dimensional Einstein tensor are

$$
\begin{gathered}
G_{00}=\frac{N^{2}}{B^{2}}\left[-3 \frac{a^{\prime \prime}}{a}+3 \frac{a^{\prime}}{a} \frac{B^{\prime}}{B}-3\left(\frac{a^{\prime}}{a}\right)^{2}\right]+3\left(\frac{\dot{a}}{a}\right)^{2}+3 \frac{\dot{a}}{a} \frac{\dot{B}}{B} \\
G_{55}=3 \frac{a^{\prime}}{a}\left(\frac{a^{\prime}}{a}+\frac{N^{\prime}}{N}\right)+3 \frac{B^{2}}{N^{2}}\left[\frac{\dot{a}}{a} \frac{\dot{N}}{N}-\left(\frac{\dot{a}}{a}\right)^{2}-\frac{\ddot{a}}{a}\right], \\
G_{05}=3 \frac{a^{\prime}}{a} \frac{\dot{B}}{B}+3 \frac{N^{\prime}}{N} \frac{\dot{a}}{a}-3 \frac{\dot{a}^{\prime}}{a} \\
G_{i i}=\frac{a^{2}}{B^{2}}\left[\frac{N^{\prime \prime}}{N}+2 \frac{a^{\prime \prime}}{a}+\left(\frac{a^{\prime}}{a}\right)^{2}+2 \frac{a^{\prime}}{a} \frac{N^{\prime}}{N}-2 \frac{a^{\prime}}{a} \frac{B^{\prime}}{B}-\frac{N^{\prime}}{N} \frac{B^{\prime}}{B}\right] \\
+\frac{a^{2}}{N^{2}}\left[-\frac{\ddot{B}}{B}-2 \frac{\ddot{a}}{a}-\left(\frac{\dot{a}}{a}\right)^{2}+2 \frac{\dot{N}}{N} \frac{\dot{a}}{a}-2 \frac{\dot{B}}{B} \frac{\dot{a}}{a}+\frac{\dot{N}}{N} \frac{\dot{B}}{B}\right]
\end{gathered}
$$

where $\dot{a} \equiv d a / d t, \ddot{a} \equiv d^{2} a / d t^{2}$ and $a^{\prime} \equiv d a / d y$. Assume that the matter in the visible brane located at the fixed plane $y=0$ takes the form of the perfect fluid, then we get $t_{N}^{M}=\delta(y) \operatorname{diag}(-\rho, p, p, p, 0) / B$. Evaluate Eq. (2) for the $G_{00}$ component Eq. (4) and the $G_{i i}$ component Eq. (7) at the boundary $y=0$, we get the junction conditions

$$
\begin{gathered}
\frac{\left[a^{\prime}\right]}{a^{0} B^{0}}=-\frac{\kappa_{5}^{2}}{3} \rho, \\
\frac{\left[N^{\prime}\right]}{N^{0} B^{0}}=\frac{\kappa_{5}^{2}}{3}(2 \rho+3 p),
\end{gathered}
$$

where superscript 0 means that the variable takes value at $y=0$ and the junction $\left[a^{\prime}\right]=a^{\prime}\left(t, 0_{+}\right)-a^{\prime}\left(t, 0_{-}\right)$. The junction of the $G_{05}$ component Eq. (6) gives the usual energy conservation equation

$$
\dot{\rho}+3 \frac{\dot{a}^{0}}{a^{0}}(\rho+p)=0 .
$$

The average of the $G_{55}$ component Eq. (5) at $y=0$ together with Eqs. (2), (8) and (9) gives

$$
\frac{1}{\left(N^{0}\right)^{2}}\left(\frac{\left(\dot{a}^{0}\right)^{2}}{\left(a^{0}\right)^{2}}-\frac{\dot{a}^{0}}{a^{0}} \frac{\dot{N}^{0}}{N^{0}}+\frac{\ddot{a}^{0}}{a^{0}}\right)=-\frac{\kappa_{5}^{2}}{3\left(B^{0}\right)^{2}} T_{55}-\frac{\kappa_{5}^{4}}{36} \rho(\rho+3 p) .
$$

In terms of the cosmic time $d \tau=N^{0} d t$, the above equation can be rewritten as

$$
\left(\frac{a_{, \tau}^{0}}{a^{0}}\right)^{2}+\frac{a_{, \tau \tau}^{0}}{a^{0}}=-\frac{\kappa_{5}^{2}}{3\left(B^{0}\right)^{2}} T_{55}-\frac{\kappa_{5}^{4}}{36} \rho(\rho+3 p) .
$$


Now if we fix the gauge $B^{0}=1$ and assume that $T_{55}$ is a constant, then we get the four dimensional Friedmann equation

$$
H^{2}=\left(\frac{a_{, \tau}^{0}}{a^{0}}\right)^{2}=\frac{\kappa_{5}^{4}}{36} \rho^{2}-\frac{\kappa_{5}^{2}}{6} T_{55}+\frac{\mathcal{C}}{\left(a^{0}\right)^{4}},
$$

where $\mathcal{C}$ is an integration constant. If our 3 -brane is embedded in a five dimensional Ads spacetime, then $T_{55}=\Lambda_{B}$ and $\rho=T+\rho_{b}$, here $-\Lambda_{B}$ is the five dimensional cosmological constant, $\rho_{b}$ is the matter density in the brane and $T$ is the brane tension. If for some reasons, we have fine-tuned $T^{2}=6 \Lambda_{B} / \kappa_{5}^{2}$ and $\mathcal{C}=0$, then from Eq. (12), we get the familiar brane cosmological equation

$$
H^{2}=\frac{\kappa_{5}^{4}}{36} \rho_{b}^{2}+\frac{\kappa_{5}^{4}}{18} T \rho_{b}
$$

If the bulk energy momentum tensor takes the perfect fluid form $T_{N}^{M}=$ $\operatorname{diag}\left(-\rho_{B}, p_{B}, p_{B}, p_{B},-\rho_{B}\right)$, then we can get the solutions to Eq. (2) as follows:

$$
\begin{gathered}
B(t, y)=1, \\
\frac{\dot{a}(t, y)}{N(t, y)}=\alpha(t), \\
a^{2}(t, y)=A(t) \cosh (\mu y)+B(t) \sinh (\mu|y|)+C(t),
\end{gathered}
$$

where $\mu=\sqrt{-2 \kappa_{5}^{2} \rho_{B} / 3}$ in the case that $\rho_{B}<0$ and $C(t)=3 \alpha^{2}(t) /\left(\kappa_{5}^{2} \rho_{B}\right)$, or

$$
a^{2}(t, y)=A(t) \cos (\mu y)+B(t) \sin (\mu|y|)+C(t),
$$

where $\mu=\sqrt{2 \kappa_{5}^{2} \rho_{B} / 3}$ in the case that $\rho_{B}>0$, or

$$
a^{2}(t, y)=\alpha^{2}(t) y^{2}+D(t)|y|+E(t),
$$

in the case that $\rho_{B}=0$. Furthermore, we can fix the temporal gauge such that $N^{0}=$ 1. The integration constants $A(t), B(t), D(t), E(t)$ and $\alpha(t)$ can be determined from the junction condition Eqs. (8) and (9).

If the bulk energy momentum tensor takes other forms, Chung and Freese argued that almost any relationship between $\rho$ and $H$ was possible in Ref. 19. To see this, we take the matter source in our brane Universe to be dust, i.e., $\rho=$ $\rho_{b} \propto\left(a^{0}\right)^{-3}$ and $p=p_{b}=0$. Then the junction condition Eqs. (8) and (9) can be rewritten as

$$
\frac{2\left[a^{\prime}\right]}{a^{0} B^{0}}=-\frac{\left[N^{\prime}\right]}{N^{0} B^{0}}=-\frac{2 \kappa_{5}^{2}}{3} \rho_{b} .
$$

If we need a relation $\rho_{b}=\nu H^{q}$, then we shall look for the solution to the following equation

$$
\frac{3\left[a^{\prime}\right]}{a^{0} B^{0}}=-\nu \kappa_{5}^{2}\left(\frac{\dot{a}^{0}}{N^{0} a^{0}}\right)^{q} .
$$

The solutions to Eqs. (18) and (19) are

$$
N(t, y)=B(t, y)=\exp (\beta(t)|y|),
$$




$$
a(t, y)=\left(\frac{t}{t_{0}}\right)^{q / 3} \exp (-\beta(t)[F(|y|)-F(y=0)] / 2),
$$

where $\beta(t)=\kappa_{5}^{2} \nu(q / 3 t)^{q} / 3, F(y)$ is any smooth function satisfying $F^{\prime}(y=0)=1$ and $t_{0}$ is an integration constant. Substitute the above solutions to Eq. (2), we can get the bulk energy momentum tensor.

\subsection{New Model}

In ref. 26, we proposed a new Friedman equation

$$
H^{2}=\sigma \rho_{b}\left[1+\exp \left(-\gamma \rho_{b}\right)\right]^{n} .
$$

In this section, we show how to derive the model. Now we are looking for the solutions to the full five dimensional Einstein equation (2) satisfying the boundary condition Eq. (18) and the following equation

$$
\frac{\dot{a}^{0}}{N^{0} a^{0}}=\sqrt{\sigma} \sqrt{\frac{-3\left[a^{\prime}\right]}{\kappa_{5}^{2} a^{0} B^{0}}}\left(1+\exp \left[\frac{3 \gamma\left[a^{\prime}\right]}{\kappa_{5}^{2} a^{0} B^{0}}\right]\right)^{n / 2} .
$$

The general solutions which satisfy Eqs. (18) and (23) are

$$
\begin{gathered}
N(t, y)=B(t, y)=\exp \left(\kappa_{5}^{2} \rho_{b}(t)|y| / 3\right), \\
a(t, y)=a^{0}(t) \exp \left(-\kappa_{5}^{2} \rho_{b}(t)[F(|y|)-F(y=0)] / 6\right),
\end{gathered}
$$

where $F(y)$ is any smooth function satisfying $F^{\prime}(y=0)=1, \rho_{b}(t) \propto\left(a^{0}\right)^{-3}$ and $a^{0}(t)$ are the solutions to Eq. (22). Substitute the above solutions into Eq. (2), we can get the five dimensional energy momentum tensor which provides the new model.

In general, the modified Friedmann equations for a spatially flat universe are

$$
\begin{gathered}
H^{2}=H_{0}^{2} g(x), \\
\frac{\ddot{a}}{a}=H_{0}^{2} g(x)-\frac{3 H_{0}^{2} x}{2} g^{\prime}(x)\left(\frac{\rho+p}{\rho}\right), \\
\dot{\rho}+3 H(\rho+p)=0,
\end{gathered}
$$

where $x=8 \pi G \rho / 3 H_{0}^{2}, 1+z=a_{0} / a$ is the redshift parameter, a subscript 0 means the value of the variable at present, $g(x)$ is a general function of $x$ and from now on $g^{\prime}(x)=d g(x) / d x$. Let $\Omega_{\mathrm{m} 0}=8 \pi G \rho_{\mathrm{m} 0} / 3 H_{0}^{2}$, then $x_{0}=\Omega_{\mathrm{m} 0}$ and $x=\Omega_{\mathrm{m} 0}(1+z)^{3}$ during matter dominated era. For our new model, we have

$$
g(x)=x\left(1+e^{-\alpha x}\right)^{n}=x\left[1+\left(x_{0}^{-1 / n}-1\right)^{x / x_{0}}\right]^{n},
$$

where $\alpha=-\ln \left(\Omega_{\mathrm{m} 0}^{-1 / n}-1\right) / \Omega_{\mathrm{m} 0}>0$.

Dark energy models have been extensively studied in the literature. Most researchers studied dark energy models by constraining the dark energy equation of 
state parameter $\omega_{\mathrm{Q}}$. To compare the modified model with the dark energy model, we make the following identification

$$
\omega_{\mathrm{Q}}=\frac{x g^{\prime}(x)-g(x)}{g(x)-x} .
$$

The transition from deceleration to acceleration happens when the deceleration parameter $q=-\ddot{a} / a H^{2}=0$. From equations (26) and (27), the transition redshift $z_{\mathrm{T}}$ is given by

$$
g\left[\Omega_{\mathrm{m} 0}\left(1+z_{\mathrm{T}}\right)^{3}\right]=\frac{3}{2} \Omega_{\mathrm{m} 0}\left(1+z_{\mathrm{T}}\right)^{3} g^{\prime}\left[\Omega_{\mathrm{m} 0}\left(1+z_{\mathrm{T}}\right)^{3}\right] .
$$

\section{Supernova fitting result}

The current age of the Universe is

$$
H_{0} t_{0}=H_{0} \int_{0}^{t_{0}} d t=\int_{0}^{\infty} \frac{d z}{(1+z) g^{1 / 2}\left[\Omega_{\mathrm{m} 0}(1+z)^{3}\right]}
$$

The luminosity distance $d_{\mathrm{L}}$ is defined as

$$
d_{\mathrm{L}}(z)=a_{0}(1+z) \int_{t}^{t_{0}} \frac{c d t^{\prime}}{a\left(t^{\prime}\right)}=\frac{c(1+z)}{H_{0}} \int_{0}^{z} g^{-1 / 2}\left[\Omega_{\mathrm{m} 0}(1+u)^{3}\right] d u .
$$

The apparent magnitude redshift relation is

$$
m(z)=M+5 \log _{10} d_{\mathrm{L}}(z)+25=\mathcal{M}+5 \log _{10} \mathcal{D}_{\mathrm{L}}(z),
$$

where $\mathcal{D}_{\mathrm{L}}(z)=H_{0} d_{\mathrm{L}}(z)$ is the "Hubble-constant-free" luminosity distance, $M$ is the absolute peak magnitude and $\mathcal{M}=M-5 \log _{10} H_{0}+25$. The parameters in our model are determined by minimizing

$$
\chi^{2}=\sum_{i} \frac{\left[m_{\mathrm{obs}}\left(z_{i}\right)-m\left(z_{i}\right)\right]^{2}}{\sigma_{i}^{2}}
$$

where $\sigma_{i}$ is the total uncertainty in the observation. The $\chi^{2}$-minimization procedure is based on MINUIT code ${ }^{27}$. The observational data used are: the 20 radio galaxy and 78 supernova data by Daly and Djorgovski ${ }^{8}$, the 172 supernova data with $z>0.01$ and $A_{v}<0.5 \mathrm{mag}$ by Tonry et al. and the 22 supernova data with $A_{v}<0.5 \mathrm{mag}$ by Barris et al. ${ }^{2}$, and the latest 157 supernova data compiled by Riess et al. ${ }^{3}$ combined with the CMB shift parameter $\mathcal{R}=\Omega_{\mathrm{m} 0}^{1 / 2} H_{0} d_{\mathrm{L}}\left(z_{\mathrm{ls}}\right) /\left(c\left(1+z_{\mathrm{ls}}\right)\right)=$ $1.716 \pm 0.062{ }^{28}$, here $z_{\mathrm{ls}}=1089 \pm 1^{5}$. For the SN Ia data compiled by Tonry et al. and Barris et al., a $500 \mathrm{~km} / \mathrm{s}$ uncertainty in quadrature to the redshift errors is added to account for the velocity uncertainty and $\chi^{2}$ is calculated by marginalizing over $H_{0}$. The marginalization is done by integrating $e^{-\chi^{2} / 2}$ over all possible values of $H_{0}$. Since $H_{0}$ is appeared linearly in $\chi^{2}$, the integration process is equivalent to minimize $\chi^{2}$ over $H_{0}$.

From equation (29), it is easy to see that at high redshift $z, x$ is very large and $g(x) \sim x$. Therefore the standard model is recovered at early times. In the 
future, $\exp (-\alpha x) \rightarrow 1$ and $g(x) \approx 2^{n} x$, so the Universe will again evolve as $t^{2 / 3}$. Substituting the new model (29) into equation (27), during the matter domination we get

$$
\frac{\ddot{a}}{a H_{0}^{2}}=-\frac{1}{2} x\left(1+e^{-\alpha x}\right)^{n}+\frac{3}{2} n \alpha x^{2}\left(1+e^{-\alpha x}\right)^{n-1} e^{-\alpha x} .
$$

As $x \gg 1$, we have $\ddot{a} /\left(a H_{0}^{2}\right) \rightarrow-x / 2$. As $x \rightarrow 0, \ddot{a} /\left(a H_{0}^{2}\right) \rightarrow 0$. In this model, equation (31) becomes

$$
1+\left(\Omega_{\mathrm{m} 0}^{-1 / n}-1\right)^{\left(1+z_{\mathrm{T}}\right)^{3}}+3 n \ln \left(\Omega_{\mathrm{m} 0}^{-1 / n}-1\right)\left(\Omega_{\mathrm{m} 0}^{-1 / n}-1\right)^{\left(1+z_{\mathrm{T}}\right)^{3}}\left(1+z_{\mathrm{T}}\right)^{3}=0
$$

If we compare this model with the dark energy model, from Eq. (30), we get

$$
\omega_{\mathrm{Q} 0}=\frac{n\left(1-\Omega_{\mathrm{m} 0}^{1 / n}\right) \ln \left(\Omega_{\mathrm{m} 0}^{-1 / n}-1\right)}{\Omega_{\mathrm{m} 0}\left(1-\Omega_{\mathrm{m} 0}\right)} .
$$

In the fit, the range of parameter space for $n$ is $(0,45]$ and the range of parameter space for $\Omega_{\mathrm{m} 0}$ is $(0,1]$. The best fit results are summarized in table 1 . In table 1 , we also list the transition redshift, the state of equation parameter $\omega_{\mathrm{Q} 0}$ of the equivalent dark energy model and the age of universe using the best fit parameters. For the last row in table 1, we fit the model to both the 157 gold sample supernova data and the CMB shift parameter. In this model, the Universe is older than that by the standard cosmology. The contour plots to different data sample are shown in Figs. 1-5.

\begin{tabular}{|c|c|c|c|c|c|c|c|c|}
\hline \multirow{2}{*}{$\begin{array}{c}\text { Data } \\
\#\end{array}$} & \multicolumn{2}{|c|}{$\Omega_{\mathrm{m} 0}$} & \multicolumn{2}{|c|}{$n$} & \multirow{2}{*}{$\chi^{2}$} & $\omega_{\mathrm{Q} 0}$ & $z_{T}$ & $H_{0} t_{0}$ \\
\cline { 2 - 9 } & $68 \%$ & $99 \%$ & $68 \%$ & $99 \%$ & & & & \\
\hline 98 & $0.33_{-0.14}^{+0.19}$ & $0.33_{-0.15}^{+0.29}$ & $2.46_{-0.34}^{+0.31}$ & $2.46_{-0.6}^{+12}$ & 88.1 & -2.27 & 0.76 & 0.97 \\
\hline 194 & $0.54_{-0.09}^{+0.06}$ & $0.54_{-0.09}^{+0.12}$ & $3.34_{-1.22}^{+7.21}$ & $3.34_{-1.67}^{+108}$ & 195.96 & -3.62 & 0.30 & 0.86 \\
\hline 158 & $0.46_{-0.11}^{+0.09}$ & $0.46_{-0.21}^{+0.15}$ & $2.60_{-0.54}^{+4.70}$ & $\geq 1.75$ & 181.4 & -2.85 & 0.44 & 0.90 \\
\hline
\end{tabular}

In Fig. 4, we plot the dimensionless acceleration $\ddot{a} / a H_{0}^{2}$ against the redshift. It is clear that the model have deceleration phase in recent past and acceleration phase at present. In Fig. 5, the evolution of $\omega_{\mathrm{Q}}$ of the equivalent dark energy model is shown for the best fit parameters to the combined supernova and CMB data. Around $z \sim 2, g(x) \approx x$ and the Universe became indistinguishable from the standard matter dominated universe.

As shown in Fig. 4, the new model gives past deceleration, current acceleration and future deceleration or near zero acceleration. From theoretical point of view, future deceleration is favored because it avoids the horizon problem. In Ref. 10, the authors show that eternal accelerating dark energy models prevent us from ever measuring inflationary perturbations which originated before the ones currently observable due to the growth of the comoving Hubble scale in the future. The Universe in this new model is older than that in standard cosmology as shown 
8

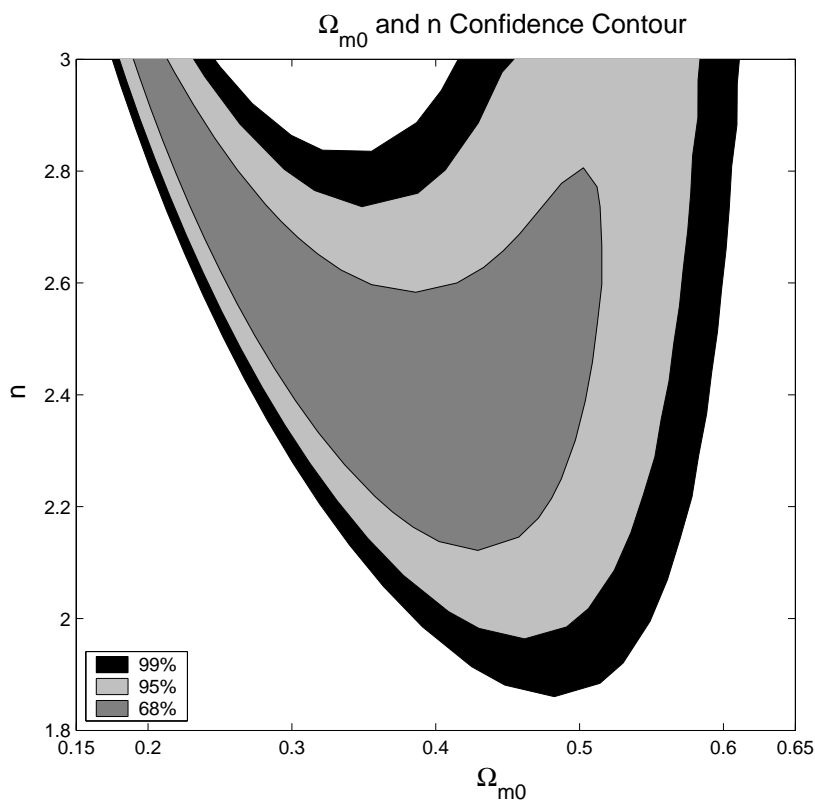

Fig. 1. The $\Omega_{\mathrm{m} 0}$ and $n$ contour plot for the 20 radio galaxy and 78 supernova data by Daly and Djorgovski

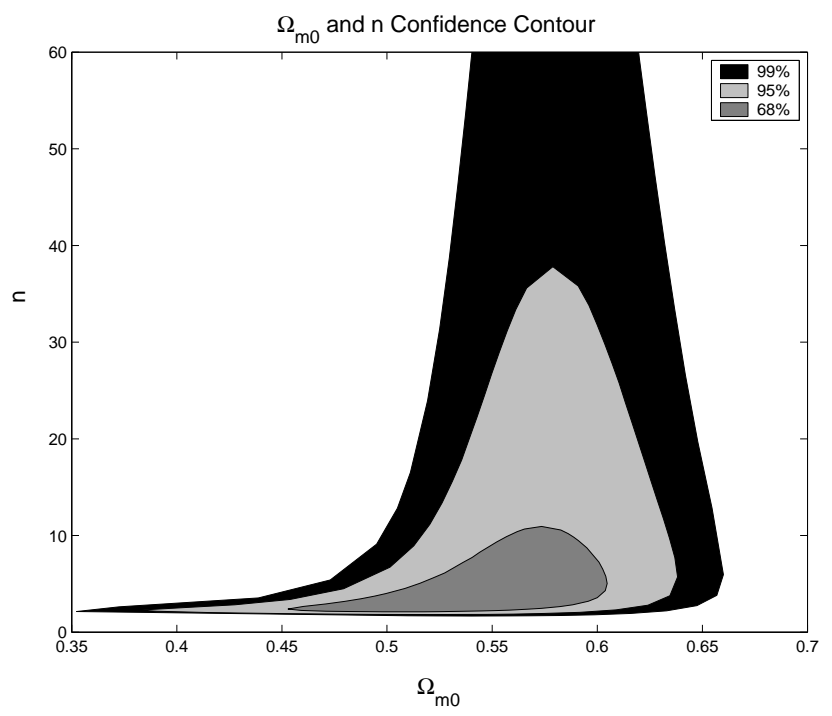

Fig. 2. The $\Omega_{\mathrm{m} 0}$ and $n$ contour plot for the 194 combined supernova data by Tonry et al. and Barris al.

in table 1. Gong and Daly and Djorgovski found the model independent result $z_{\mathrm{T}} \sim 0.4^{8}$. The result is consistent with ours as seen from table 1 . The somewhat 


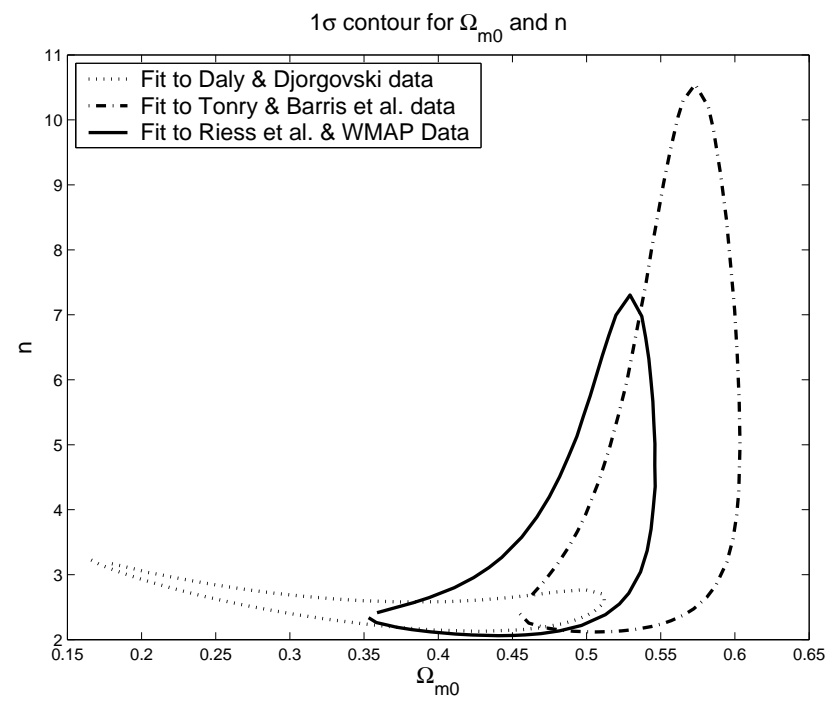

Fig. 3. The $1 \sigma \Omega_{\mathrm{m} 0}$ and $n$ contour plots

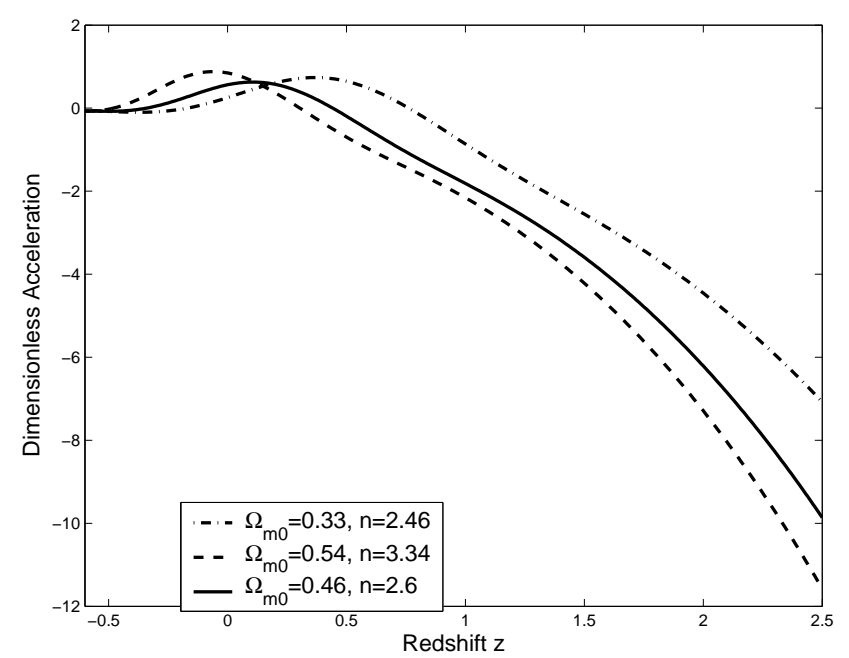

Fig. 4. $\ddot{a} / a H_{0}^{2}$ versus the redshift $z$ for the best fit parameters listed in Table I.

model independent study by Alam, Sahni, Saini and Starobinsky found that $\omega_{\mathrm{Q}}$ evolves rapidly from zero at high $z$ to a strongly negative value at present ${ }^{14}$. This result is also consistent with ours as shown in table 1 and Fig. 5. In conclusion, we proposed a new model which is consistent with current supernova observations and avoids the future horizon problem. It is difficult to discriminate this model from dark energy models with current observations. Future supernova data from the dedicated satellite telescope (such as Supernova/Acceleration Probe) is necessary 
10

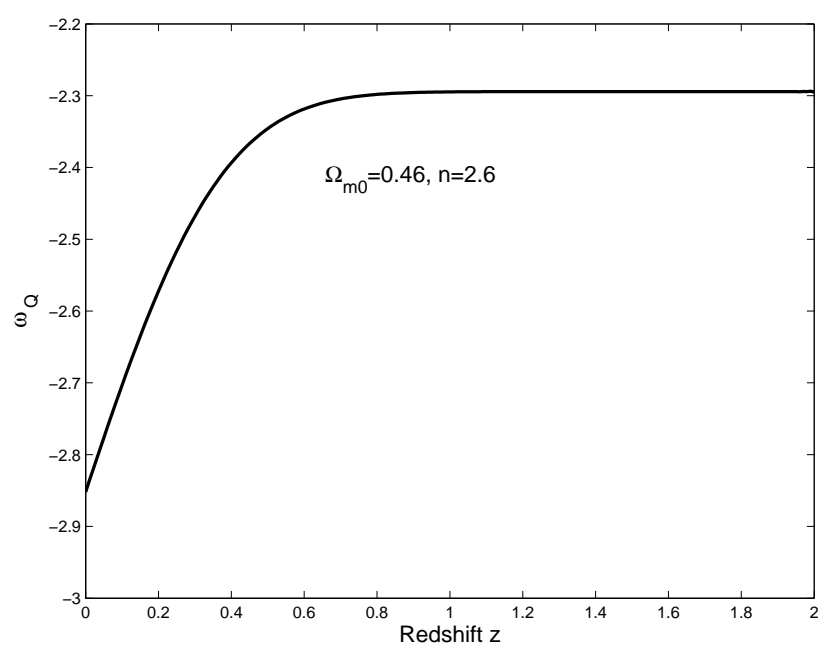

Fig. 5. $\omega_{\mathrm{Q}}$ versus the redshift $\mathrm{z}$ for the best fit parameters $\Omega_{\mathrm{m} 0}=0.52$ and $n=2.8$.

to differentiate these models.

\section{acknowledgments}

The work is supported by Chongqing University of Post and Telecommunication under grants A2003-54 and A2004-05.

\section{References}

1. S. Perlmutter et al., Astrophys. J. 517, 565 (1999); P.M. Garnavich et al., Astrophys. J. Lett. 493, L53 (1998); A.G. Riess et al., Astron. J. 116, 1009 (1998).

2. J.L. Tonry et al., Astrophys. J. 594, 1 (2003); B.J. Barris et al., Astrophys. J. 602, $571(2004)$.

3. A. G. Riess et al., Astrophys. J. 607, 665 (2004).

4. P. de Bernardis et al., Nature 404, 955 (2000); Hanany S et al., Astrophys. J. Lett. 545, L5 (2000).

5. C.L. Bennett et al., Astrophys. J. Supp. Ser. 148, 1 (2003); D.N. Spergel et al., Astrophys. J. Supp. Ser. 148, 175 (2003).

6. A.G. Riess, Astrophys. J. 560, 49 (2001).

7. M.S. Turner and A.G. Riess, Astrophys. J. 569, 18 (2002).

8. R.A. Daly and S.G. Djorgovski, Astrophys. J. 597, 9 (2003); astro-ph/0403664; Y. Gong, Int. J. Mod. Phys. D in press, astro-ph/0402512.

9. A. Cohen, D. Kaplan and A. Nelson, Phys. Rev. Lett. 82 (1999) 4971; P. Hořava and D. Minic, Phys. Rev. Lett. 85 (2000) 1610; S. Thomas, Phys. Rev. Lett. 89 (2002) 081301; S.D.H. Hsu, Phys. Lett. B 594 (2004) 13; M. Li, hep-th/0403127; Q-G. Huang and M. Li, JCAP 0408, 013 (2004); hep-th/0410095; K. Ke and M. Li, hep-th/0407056; B. Wang, E. Abdalla and R.K. Su, hep-th/0404057; R.G. Cai, JCAP 0402 (2004) 007; Q-G. Huang and Y. Gong, JCAP 0408, 006 (2004); Y. Gong, Phys. Rev. D 70, 064029 (2004).

10. N. Kaloper, M. Kleban and L. Sorbo, astro-ph/0406099. 
11. R.R. Caldwell, R. Dave and P.J. Steinhardt, Phys. Rev. Lett. 80, 1582 (1998); I. Zlatev, L. Wang and P.J. Steinhardt, ibid. 82, 896 (1999); P.G. Ferreira and M. Joyce, Phys. Rev. Lett. 79, 4740 (1997); B. Ratra and P.J.E. Peebles, Phys. Rev. D 37, 3406 (1988); C. Wetterich, Nucl. Phys. B 302, 668 (1988); S. Perlmutter, M.S. Turner and M. White, Phys. Rev. Lett. 83, 670 (1999).

12. V. Sahni and A.A. Starobinsky, Int. J. Mod. Phys. D 9, 373 (2000); C. Rubano and J.D. Barrow, Phys. Rev. D 64, 127301 (2001); V.B. Johri, Class. Quantum Grav. 19, 5959 (2001); E. Di Pietro and J. Demaret, Int. J. Mod. Phys. D 10, 231 (2001); L.A. Ureña-López and T. Matos, Phys. Rev. D 62, 081302 (2000).

13. A.A. Sen and S. Sethi, Phys. Lett. B 532, 159 (2002); Y. Gong, Class. Quantum Grav. 194537 (2002).

14. U. Alam, V. Sahni, T.D. Saini and A.A. Starobinsky, astro-ph/0311364; U. Alam, V. Sahni and A.A. Starobinsky, JCAP 0406, 008 (2004).

15. R.R. Caldwell, Phys. Lett. B 545, 23 (2002) ; J.S. Alcaniz, astro-ph/0312424 ; M. Kaplinghat and S. Bridle, astro-ph/0312430.

16. A. Kamenshchik, U. Moschella and V. Pasquier, Phys. Lett. B 511, 265 (2001); N. Bilic, G.G. Tupper and R.D. Viollier, ibid. 535, 17 (2002); M.C. Bento, O. Bertolami and A.A. Sen, Phys. Rev. D 66, 043507 (2002); D. Carturan and F. Finelli, ibid. 68, 103501 (2003); L. Amendola, F. Finelli, C. Burigana and D. Carturan, J. Cosmology Astroparticle Phys. 0307, 005 (2003); J.V. Cunha, J.S. Alcaniz and J.A.S. Lima, Phys. Rev. D 69, 083501 (2004); Y. Gong, astro-ph/0411253.

17. C. Armendariz-Picon, T. Damour and V. Mukhanov, Phys. Lett. B 458, 209 (1999) ; T. Padmanabhan and T.R. Choudhury, Phys. Rev. D 66, 081301 (2002) ; J.S. Bagla, H.K. Jassal and T. Padmanabhan, ibid. 67, 063504 (2003); T. Padmanabhan, Phys. Rep. 380, 235 (2003); T. Padmanabhan and T.R. Choudhury, Mon. Not. Roy. Astron. Soc. 344, 823 (2003); L.P. Chimento, Phys. Rev. D 69, 123517 (2004); A. Sen, JHEP 0204, 048 (2002); P.S. Piao, R.G. Cai and Y.Z. Zhang, Phys. Rev. D 66, 121301 (2002).

18. P. Binétruy, C. Deffayet and D. Langlois, Nucl. Phys. B 565, 269 (2000).

19. D.J. Chung and K. Freese, Phys. Rev. D 61, 023511 (1999).

20. K. Freese and M. Lewis, Phys. Lett. B 540, 1 (2002); K. Freese, Nucl. Phys. Suppl. Ser. 124, 50 (2003); P. Gondolo and K. Freese, Phys. Rev. D 68, 063509 (2003).

21. G.R. Dvali, G. Gabadadze and M. Porrati, Phys. Lett. B 485, 208 (2000); C. Deffayet, ibid. 502, 199 (2001); C. Deffayet, G.R. Dvali and G. Gabadadze, Phys. Rev. D 65, 044023 (2002); G.R. Dvali and M. Turner, astro-ph/0301510; A. Lue, R. Scoccimarro and G. Starkman , Phys. Rev. D 69, 044005 (2004).

22. S. Sen and A.A. Sen, Astrophys. J. 588, 1 (2003) ; A.A. Sen and S. Sen, Phys. Rev. D 68, 023513 (2003); M. Szydlowski and W. Czaja, astro-ph/0309191; W. Godlowski, M. Szydlowski and A. Kraweic, astro-ph/0309569.

23. Z.H. Zhu and M. Fujimoto, Astrophys. J. 581, 1 (2002); 585, 52 (2003); Astrophys. J. 602, 12 (2004); Z.H. Zhu , M. Fujimoto and X.T. He, Astrophys. J. 603, 365 (2004).

24. Y. Wang, K. Freese, P. Gondolo and M. Lewis, Astrophys. J. 594, 25 (2003); T. Multamaki, E. Gaztanaga and M. Manera, Mon. Not. Roy. Astron. Soc. 344, 761 (2003); A. Dev, J.S. Alcaniz and D. Jain, astro-ph/0305068; W.J. Frith, Mon. Not. Roy. Astron. Soc. 348, 916 (2004); S. Nesseris and L. Perivolaropoulos, astro-ph/0401556.

25. Y. Gong and C.K. Duan, Class. Quantum Grav. 21, 3655 (2004); Mon. Not. Roy. Astron. Soc. 352, 847 (2004).

26. Y. Gong, X.M. Chen and C.K. Duan, Mod. Phys. Lett. A 19, 1933 (2004).

27. Minuit 2002, http://wwwasd.web.cern.ch/wwwasd/cernlib 
December 10, 2018 1:44 WSPC/INSTRUCTION FILE altnew

/download/2002_source/tar/minuit32_src.tar.gz.

28. J.R. Bond, G. Efstathiou and M. Tegmark, Mon. Not. Roy. Astron. Soc. 291, L33 (1997); A. Melchiorri, L. Mersini, C.J. Ödman and M. Trodden, Phys. Rev. D 68, 043509 (2003); Y. Wang and P. Mukherjee, Astrophys. J. 606, 654 (2004); Y. Wang and M. Tegmark, Phys. Rev. Lett. 92, 241302 (2004). 